\title{
Cultura, religión y humanismo en el pensamiento de T. S. Eliot
}

\section{Culture, religion and humanism in T. S. Eliot's thought}

\author{
Pablo Zambrano CARBALlo \\ Universidad de Huelva \\ zambrano@uhu.es
}

Recibido: 06-09-2010

Aceptado: 09-12-2010

\section{Resumen}

El pensamiento sociocultural del influyente poeta y crítico T. S. Eliot es la parte de su obra que menos atención crítica ha merecido pero que, al mismo tiempo, más ha influido en las últimas décadas en el progresivo desprestigio que, desde presupuestos ideológicos, ha sufrido el conjunto de su legado. Sin obviar sus muchos elementos discutibles, muy polémicos ya desde su formulación a partir de la tercera década del siglo pasado e incluso desfasados desde la perspectiva actual, este artículo revisa las principales líneas de la teoría sociocultural eliotiana, rescata los temas que mantienen su presencia en el debate actual y se centra con detalle en las relaciones entre cultura, religión y humanismo, determinantes para el esclarecimiento de muchas de sus ambigüedades.

Palabras clave: Babbitt, Cultura, Educación, Eliot, Europa, Humanismo, Maritain, Religión.

\begin{abstract}
T. S. Eliot's sociocultural thought has traditionally deserved less critical attention than his labour as a poet and literary critic. However, over the last decades, his
\end{abstract}


role as one of Europe's most respected and influential intellectuals has been widely attacked on ideological basis derived from his controversial ideas on social and cultural matters. This article aims at a reexamination of Eliot's sociocultural proposals, focusing mainly on the links between culture, religion and humanism, in order to show that, although some of his polemical ideas may be well behind the times today, some others are still re-levant for the contemporary debate.

Keywords: Babbitt, Culture, Education, Eliot, Europe, Humanism, Maritain, Religion.

\section{Introducción}

La aparición en 2009 del segundo volumen de las cartas de T. S. Eliot, que cubre un periodo corto pero crucial de su vida (1923-1925), debería haber supuesto un acontecimiento cultural de primera fila, tras más de veinte años de espera desde la publicación del primer volumen en $1988 .{ }^{1} \mathrm{Si}$ el epistolario de entonces culminaba con la aparición de The Waste Land, las cartas publicadas ahora abarcan los años posteriores a la irrupción de Eliot como uno de los poetas y críticos más influyentes y controvertidos del siglo XX. De su lectura emana una figura muy compleja, empeñada no sólo en abrir nuevos caminos poéticos y críticos, sino también profundamente implicada en los principales debates culturales, políticos y sociales de la intelectualidad europea de aquellos años, como bien demostró su revista The Criterion, una implicación que se mantendría con fuerza en décadas posteriores, hasta su muerte en 1965.

$\mathrm{Y}$, sin embargo, la publicación de este nuevo volumen de cartas no ha causado el impacto cultural esperable de un escritor que desde 1922 ha venido ocupando una posición central en el canon literario y cultural anglosajón y europeo del siglo XX. $\mathrm{Y}$ es que la figura de T. S. Eliot en estos primeros años del nuevo siglo ha comenzado a desdibujarse por causas diversas que vienen actuando desde hace ya mucho tiempo, que no siempre hacen justicia al valor de su obra y que comienzan a desplazarlo, si no a la periferia del canon, sí al menos de esa posición canónica central de la que ha gozado. Vanheste resume acertadamente cómo, del estatus de poeta de culto e icono de la alta cultura en que crítica y público lo situaron hasta su muerte, Eliot se fue convirtiendo en décadas posteriores en símbolo de elitismo y conservadurismo, tachado incluso de fascista y antisemita. A su juicio, la situación a día de hoy es que "the Eliot research has become politicized, mainly focusing on social,

1 The Letters of T. S. Eliot. Vol. 1 (1898-1922). Valerie Eliot ed. San Diego-New York-London: Harcourt Brace Jovanovich, 1988; The Letters of T. S. Eliot. Vol. 2 (1923-1925). Valerie Eliot and Hugh Haughton eds. London: Faber and Faber, 2009. 
political and ideological issues rather than on his creative achievement (...) So we may conclude that little is left today of the great poet and critic that Eliot was once held to be. ${ }^{2}$ Esta rotunda afirmación quizás peque de exagerada, pero es muy sintomática de la cuanto menos tambaleante posición en que se encuentran hoy en día el legado y la figura de T. S. Eliot. Sin embargo, cuesta aceptar que una obra que contiene poemas tan emblemáticos y revolucionarios como The Waste Land o Four Quartets y ensayos tan influyentes y citados como "Tradition and the Individual Talent" o "Hamlet and his Problems" esté quedando subsumida en el magma de una deconstrucción global basada más, como bien apunta Vanheste, en razones puramente ideológicas que en argumentos artísticos. Es justo esa preponderancia de los argumentos ideológicos en el juicio de la obra de Eliot la que explica la necesidad de llevar a cabo una lectura actual y atenta de su pensamiento sociocultural, una parte de su obra que tradicionalmente ha estado a la sombra de su aclamada labor como poeta y crítico literario y que, por ello, ha recibido menos atención, pero que, de manera paradójica, ha acabado imponiéndose hoy en día como factor esencial en la evaluación del conjunto de su obra.

El objetivo principal de este artículo es la reevaluación crítica de algunos de los principales y polémicos aspectos de la compleja teoría cultural de Eliot y, más en concreto, de su particular filiación a los presupuestos del humanismo, pues consideramos que el esclarecimiento de la cuestión humanista en Eliot y su contraste particular con las ideas de Irving Babbitt y Jacques Maritain, entre otros, ayudan a poner en su debido lugar muchos de los conceptos más controvertidos de su pensamiento, algunos de los cuales son precisamente los que han venido contribuyendo con especial énfasis al desprestigio paulatino de su legado. Además, dicha reevaluación permite situar las ideas de Eliot y de otros intelectuales de la época en las coordenadas de un debate -el del papel social y cultural del humanismo- que, a tenor de lo que se publica y discute dentro y fuera el ámbito académico, sigue estando en el centro de la discusión contemporánea.

\section{El pensamiento sociocultural de Eliot}

Existe gran consenso crítico en que con la publicación en 1922 de una obra capital como The Waste Land se cierra con meridiana claridad un periodo de la producción poética y también vital de T. S. Eliot. Sin duda, el inicio definitivo de la nueva etapa lo marca su conversión al anglicanismo en 1927. La perspectiva temporal y el conocimiento más detallado que hoy tenemos de la vida de Eliot gracias a estudios señeros como los de Ackroyd, Asher, Chinitz, Gordon, Margolis o Miller ${ }^{3}$

\footnotetext{
2 J. Vanheste. Guardians of the Humanist Legacy. Leiden-Boston: Brill, 2007, p. 5.

3 P. Ackroyd. T. S. Eliot. London: Penguin, 1994 (1984); K. Ashner. T. S. Eliot and Ideology. Cambridge: Cambridge University Press, 1998 (1995); D. Chinitz. T. S. Eliot and the Cultural Divide.
} 
nos confirman en la idea de que tal conversión no fue sólo la salida a una desesperada situación anímica y espiritual sino también una decisión de honda raíz cultural. En cualquier caso, el nuevo rumbo tuvo consecuencias en su labor poética y crítica. Si el plano literario estuvo marcado por la irrupción de una poesía y un teatro de corte marcadamente confesional, el plano crítico empezó a destacar por su apología de una crítica literaria sustentada en los valores cristianos y, de modo más palmario, por su atención, creciente a lo largo de las décadas posteriores, a la elaboración de una teoría sociocultural que constituye la espina dorsal de su pensamiento y que fue tomando cuerpo en diversos ensayos para compendiarse especialmente en The Idea of a Christian Society (1939) y, sobre todo, en Notes towards the Definition of Culture (1948). ${ }^{4}$

Eliot concibe la cultura como el elemento definidor de la organización política, social, educativa y religiosa de un pueblo. Su visión de la misma es orgánica, en tanto que la define no como la acumulación de actividades diversas y valores independientes sino como la forma global de vida del conjunto de una sociedad, una forma de vida determinada sobre todo por la comunicación interactiva de los diversos grupos sociales -o clases, en la terminología eliotiana-, por una variedad de niveles de conciencia cultural y, en especial, por el nexo con el pasado, un elemento éste que se revela esencial y del que se deduce el papel primordial que Eliot asigna al mantenimiento de factores como la familia, la religión y la tradición. Muy a la contra de las muchas corrientes sociopolíticas que por la época defendían los intereses y valores de la sociedad de masas, la planificación de la cultura y, en definitiva, el dirigismo, Eliot abogó en todo momento -no sin polémica ya entonces- por el mantenimiento de las clases sociales y de las élites intelectuales y defendió su importancia como transmisores del legado cultural, algo que él juzgaba esencial para el avance de la sociedad.5

Chicago: The University of Chicago Press, 2003; L. Gordon. T. S. Eliot. An Imperfect Life. London: Faber and Faber, 1998 y Eliot's Early Years. Oxford: Oxford University Press, 1977; J. Margolis. T. S. Eliot's Intellectual Development 1922-1939. Chicago: The University of Chicago Press, 1972; J. E. Miller. T. S. Eliot. The Making of an American Poet (1888-1922). Pennsylvania: The Pennsylvania State University Press, 2005.

4 T. S. Eliot. The Idea of a Christian Society. London: Faber and Faber, 1982 (1939); Notes towards the Definition of Culture. London: Faber and Faber, 1962 (1948).

5 R. Kojecky. T. S. Eliot's Social Criticism. London: Faber and Faber, 1971, pp. 207 y 210. Tanto en la concepción orgánica de la cultura como en la teoría de las élites sociales se puede establecer un paralelismo interesante, con sus semejanzas y diferencias obvias, entre el ideario de Eliot y el de Ortega y Gasset, cuyas respectivas revistas, The Criterion y Revista de Occidente, mantuvieron durante años un fructífero intercambio. Así, Ortega y Gasset aplica también la metáfora orgánica en su idea de España como integración de partes diversas que funcionan como un organismo vivo; la cultura es precisamente la suma de todas esas funciones vitales. La enfermedad que él diagnostica se debe justo a una falta de minorías intelectuales que guíen a la sociedad: "La recomendación incluye un componente orgánico, la necesidad de propiciar un enorme apetito, y un componente emanado de su método formal, el imperativo de la selección" (H. Van Ree. Ortega y el humanismo moderno. Zaragoza: Universidad de Zaragoza, 1997, p. 99). 
Un concepto axial del pensamiento de Eliot es la estrecha vinculación entre cultura y religión, y entre ésta y el cristianismo, representado a su vez por el anglocatolicismo. El planteamiento que Eliot hace de la situación cultural europea, y que ocupa un lugar destacado en el conjunto de su pensamiento, parte de la convicción de que, con el avance del siglo XX, el viejo continente estaba siendo víctima de un proceso creciente y devastador de secularización y de desintegración cultural que para él se manifestaba sobre todo en la progresiva pérdida de comunicación entre las diversas clases sociales, en el deterioro alarmante de los niveles de calidad culturales y en la erosión que sufría la alta cultura, en la creciente influencia -o rebelión, por decirlo en clave orteguiana- de la "cultura" de masas -que él se encargaba muy bien de distinguir de la valiosa cultura popular- y, de modo muy señalado, en la obsesión de la modernidad por la separación radical entre cultura y religión, cuyo vínculo esencial era para Eliot garante precisamente de la supervivencia de la cultura europea. Ésta aparece así arropada por un cristianismo que proporciona la sangre vital al organismo que para él era Europa. El proceso de desintegración sólo podría detenerse por medio de un restablecimiento de la indisolubilidad del vínculo entre religión y cultura. En este sentido, acudiendo a argumentos antropológicos, Eliot señala que esa unidad era consustancial a los pueblos primitivos y que, con el paso del tiempo, se fue tendiendo a una diferenciación cada vez más aguda hasta desembocar incluso en la abierta oposición entre religión y cultura que él detectaba en su época. Desde su perspectiva, la supervivencia de la tradición europea sólo podría producirse dentro del marco proporcionado por el cristianismo. ${ }^{6}$

El vínculo de esencialidad entre religión y cultura es el principal cimiento sobre el que Eliot construye su teoría sociocultural. El ya mencionado Notes towards the Definition of Culture constituye el texto de referencia ineludible. Una de las ideas de partida es que el avance cultural requiere de tres condiciones fundamentales. La primera es el desarrollo orgánico de la sociedad, de modo que se favorezca la transmisión cultural, para lo cual Eliot aboga por el mantenimiento consciente de una estructura de clases sociales, algo sin duda polémico que ha conducido a su desprestigio por antidemocrático, pero que él justificó siempre desde presupuestos exclusivamente culturales y no tanto políticos o de privilegios determinados. La segunda condición, que apunta a que toda cultura debe permitir e incluso favorecer la fragmentación geográfica, hace aflorar el asunto peliagudo del regionalismo, un

6 El proceso del que apocalípticamente se quejaba Eliot sigue ocupando sin duda en la actualidad una posición central en el debate intelectual sobre el modelo de sociedad español y europeo. Lo demuestra, por ejemplo, la agria polémica hace unos años acerca de la mención, en el frustrado proyecto de Constitución Europea, a la herencia cristiana del continente, una polémica ligada, entre otros factores, a la creciente presencia e importancia del islam. No obstante, no resulta difícil el rastreo de la impronta cristiana en muchos de los valores de la sociedad europea secularizada, que en bastantes casos no son sino valores cristianos reformulados, como bien señalan V. Camps y A. Valcárcel (Hablemos de Dios. Madrid: Taurus, 2007). 
concepto que Eliot, pese a lo sorprendente que pueda resultar en principio, ve con buenos ojos. La última condición señala la relación de equilibrio que debe regir la tensión entre la unidad y la diversidad religiosas, es decir, la universalidad doctrinal compatible con la particularidad de culto y devoción. Del cumplimiento razonable de estas tres condiciones depende en gran medida el avance o el retroceso de una cultura determinada. En tal sentido, puede afirmarse que el pesimismo casi apocalíptico de Eliot sobre el futuro cultural de Europa arranca, en buena medida, de su constatación de que tales condiciones se habían dejado de cumplir total o parcialmente, algo que, a sus ojos, estaba conduciendo a la destrucción cultural del continente. Por el contrario, la salud y el florecimiento de la cultura europea era una tarea que implicaba a todos los ciudadanos, en tanto que, como círculos concéntricos, la cultura del individuo se vincula siempre a la de su clase y ésta, a su vez, a la del conjunto de la sociedad. El relevante papel que Eliot concede a los tres niveles de cultura mencionados -el individual, el del grupo y el de la sociedad- explica la cerrada apología que hace del mantenimiento de las clases sociales y de las élites intelectuales. Es inevitable que, desde nuestra perspectiva actual, el ideario eliotiano sobre la organización social y sus reservas acerca del igualitarismo resulten aún más polémicas y hasta difíciles de digerir que cuando se formularon. Conviene, por tanto, hacer unas consideraciones más detenidas sobre el asunto.

En el esquema social que Eliot plantea, los efectos negativos sobre la cultura de la desaparición de las clases serían irreparables, ya que éstas cumplen sobre todo un papel determinante: el de preservar y transmitir a las generaciones siguientes la parcela de la cultura general que les pertenece. Eliot se cuida mucho de subrayar que el mantenimiento y la transmisión de las parcelas culturales propias redundan en beneficio no sólo de la clase social encargada de cada una de ellas sino de toda la sociedad. Si se tiene en cuenta que Eliot formula sus teorías en el contexto de un continente que, en general, tendía a la abolición de las clases sociales, al menos tal y como éstas se habían venido concibiendo, no extraña que su pensamiento se tiña de utopía social cuando plantea, por ejemplo, la oportunidad de crear grupos organizados compuestos por los ciudadanos más preparados para que, cada uno con el poder de decisión que les corresponda, velen por el florecimiento de las artes, la ciencia, la política, la filosofía, etc. Constituirían lo que Eliot denomina élites y su creación estaría encaminada a un ambicioso bien: que todos los puestos de la sociedad, desde el más humilde al más elevado, estén ocupados por aquellos individuos que posean el mayor nivel de capacitación que cada puesto requiera. Eliot no plantea su teoría de las élites en el vacío. Independientemente de las matizaciones importantes que puedan hacerse, y que caen fuera del objetivo de este estudio, la teoría eliotiana cuenta con claros precedentes, por ejemplo, en Coleridge, para quien la transmisión del legado cultural correspondía a la parte más educada y preparada de la sociedad, que él llamaba "the clerisy", conectada obviamente con la 
Iglesia pero con una vocación de servicio a toda la sociedad. También Matthew Arnold defiende en su célebre Culture and Anarchy (1869) 7 que el orden necesario para acabar con la anarquía anticultural del materialismo y del liberalismo sólo vendrá de una aristocracia intelectual compuesta por ciudadanos de todas las clases sociales que, abandonando cada uno de ellos su espíritu de clase particular, se vuelquen en el bien común. No menos importante para su teoría de las élites es el concepto de los clercs -la parte intelectual y educada de la sociedad- que Eliot recogió de ciertos críticos franceses, sobre todo de Julien Benda. ${ }^{8}$

Desde la sociedad democrática actual, el planteamiento eliotiano de las élites y su firme apología de las clases sociales resultan cuanto menos incómodos y difíciles de aceptar sin más. En este punto es conveniente, sin embargo, no dejarse llevar por los propios planteamientos, analizar con cuidado lo que Eliot expone, tener en cuenta la época y el contexto en el que se sitúan sus ideas y, en todo caso, saber espigar con objetividad entre las mismas. Así, para situar el asunto en su justo punto, no hay que olvidar que, como ya ha quedado apuntado, Eliot concibe la cultura no como una heterogeneidad de creencias y actividades sino como una forma de vida que orgánicamente es alimentada por el conjunto de la sociedad. Ello significa que todos los individuos participan de la generación de cultura, aunque no de las mismas actividades ni en igual grado. Estamos por tanto ante una organización social definida, de arriba abajo, por una gradación de diferentes niveles culturales que han de mantener una interconexión vital para la supervivencia de cada uno de ellos. Eliot, con cierto temor quizás a que sus ideas se malinterpreten, se cubre en buena medida las espaldas cuando subraya que, de ningún modo, de la organización social que él plantea deba inferirse la conclusión de que las clases sociales superiores en la escala tengan más (la cursiva es de Eliot) cultura que las inferiores, sino que son depositarias de una mayor conciencia y especialización culturales. En este punto, resulta esclarecedora la comparación de las ideas de Eliot acerca de la cultura, las clases y las élites con las del influyente Karl Mannheim, para subrayar diferencias de calado. Mientras que Eliot analiza la cultura como fruto de toda la sociedad en su conjunto y aboga por una sola élite unida en su diversidad, para Mannheim la cultura es creación de un conjunto de élites que se ven amenazadas por la participación en las actividades culturales de una parte cada vez mayor de la población. Si se entiende bien lo que Eliot plantea y consigue verse la cuestión sin los recelos que inevitablemente es fácil que afloren en este punto, lo cierto es que se deshace con facilidad el extendido prejuicio del rechazo e incluso el desprecio de Eliot por la cultura popular. Es más, lo que sí queda claro en su teoría es que la alta cultura, por cuya preservación tanto luchó, aparece como sublimación de la popular, cuya preservación es, por tanto, igual de necesaria. Por el contrario, el desprecio por la cul-

\footnotetext{
7 Matthew Arnold. Culture and Anarchy. Oxford: Oxford University Press, 2006 (1869).
}

8 Vid. R. Kojecky. op. cit. passim y, en concreto, pp. 19-34; y J. Vanheste. op. cit. passim. 
tura de masas es evidente, pues Eliot relaciona su tendencia a la uniformidad y a la anulación de la gradación mencionada con la muerte de la cultura verdadera, tanto de la alta como de la popular. Tienen razón los que lo consideran un autor elitista: lo es, pero eso no debe justificar que se rechacen sus argumentos en función sólo de un análisis parcial de sus teorías. La premisa de que la cultura, entendida del modo en que él la entiende, constituye el cimiento principal de la sociedad lo lleva al convencimiento de que la construcción de la democracia -en la que, sin ninguna duda, creía- exige la existencia de niveles culturales diversos, que irremediablemente son al mismo tiempo niveles de poder en cuanto que una parte más reducida de la sociedad, pero poseedora de una conciencia y especialización culturales más elevadas, ejerce el mismo poder que otra parte más extensa pero con un nivel de cultura menos consciente y especializado. De todas formas, el anacronismo de la teoría eliotiana de las élites -que no su polémica- es sólo aparente, pues, con todas las matizaciones que sin duda pueden plantearse desde ámbitos muy diversos, el de las élites y su problemática integración en el sistema democrático sigue siendo un asunto capital en una época de globalización económica, política y mediática en la que la teoría del hombre-masa orteguiano no parece haber perdido un ápice de vigencia, como bien demuestra el planteamiento de Tedesco de que "si bien en una sociedad democrática la distinción entre los miembros de las élites dirigentes y el resto de la ciudadanía es una distinción dinámica, lo cierto es que la responsabilidad por las decisiones es mucho mayor en aquellos que manejan áreas más sensibles desde el punto de vista de las consecuencias de sus decisiones: los científicos, los dirigentes políticos, los dirigentes empresarios". 9

La importancia capital de las clases sociales se debe en gran medida a que Eliot las concibe como el pilar esencial sobre el que se apoya una verdadera comunidad cultural, que proviene de un equilibrio no siempre fácil de conseguir entre unidad y diversidad. Es precisamente esta concepción orgánica la que le permite justificar la tensión, para él absolutamente necesaria, que debe producirse entre la unidad cultural esencial para todo país y la influencia e incluso presión que sobre dicha unidad deben ejercer el regionalismo y el localismo, entendiéndolos no como la defensa egoísta y numantina de unos objetivos particulares y mezquinos, sino como aportación al beneficio conjunto. La clave para el florecimiento cultural está en conseguir un punto de equilibrio en dicha tensión. Teniendo esto en cuenta, y en contra de los que descartan sin más los argumentos de Eliot como absolutamente reaccionarios, puede reivindicarse justo lo contrario: que, correctamente interpretadas y más allá de lo acertado de la terminología utilizada por Eliot, sus ideas se acercan más al concepto antropológico moderno de "inculturación", entendido como "la potenciación de los valores universales de una cultura particular, sin dañar su pro-

9 J. C. Tedesco. "Humanismo y educación". En M. L. Amigo y M. Cuenca eds. Humanismo y valores. Bilbao: Universidad de Deusto, 2003, 377-388, p. 387. 
pia identidad singular (...), una acción cultural exterior que no destruye el interior de la cultura particular, sino que potencia en ella la dimensión de universalidad intercultural, a menudo oculta por la afirmación identitaria de su singularidad", que al de "aculturación" como "dominación cultural que produce la consiguiente deculturación de la cultura dominada". ${ }^{10}$ Eliot no plantea la resurrección artificial de culturas ya extinguidas, ni el mantenimiento a toda costa de una cultura arcaica en condiciones modernas, sino una renovación cultural que, desde la conciencia de la tradición, haga posible una cultura contemporánea vital. Teniendo en cuenta que una de las ideas axiales del pensamiento eliotiano es que la construcción sólida del presente y del futuro sólo puede realizarse desde una conciencia y asimilación creativas del pasado, el mantenimiento y el fomento de lo que él llama "culturas satélites" -aquellas que, por razones diversas, establecen una relación con otra más poderosa- deben estar encaminados a preservar sus características culturales propias y la sintonía de las mismas con otras culturas próximas, en aras siempre del enriquecimiento y el desarrollo mutuos. Se explica así que Europa y la diversidad de pueblos que la forman sean semejantes en el planteamiento eliotiano a una constelación de culturas recorridas todas ellas por la espina dorsal de la conciencia de pertenecer a una tradición común.

No obstante, la unidad cultural necesaria en la base de toda sociedad debe ser compatible no sólo con la diversidad, sino con una fricción entre los distintos sectores culturales que configuran tal diversidad -sean éstos las clases o las regiones-, pues Eliot la contempla como un factor de creatividad y desarrollo: "La generalización de la irritación es la mejor garantía de paz", afirma tajante. ${ }^{11}$ Esta teoría de la fricción cultural, pese a que data de 1948 y a que algunos de sus principios han quedado claramente obsoletos, es uno de los elementos del pensamiento eliotiano que, mutatis mutandi, puede tener un acomodo más fácil en el contexto general de la Europa y, en concreto, de la España de hoy. Son significativos a este respecto los casos de Alemania e Italia, que Eliot toma de ejemplos de cómo dos naciones fundamentales para la configuración del continente se han desarrollado históricamente en una continua situación de marcado regionalismo sin el que, sin embargo, no se entendería el total de sus respectivas culturas. Cualquier tendencia a maquillar dicha historia de regionalismo con el objetivo encubierto de imponer una lealtad única a una supraentidad nacional causaría un daño enorme a la cultura tradicional, la única con capacidad de conseguir la renovación de la sociedad.

10 A. Aguirre. "Humanismo e inculturación", en M. L. Amigo ed. Humanismo para el siglo XXI. Bilbao: Universidad de Deusto, 2003, 393-399, pp. 396 y 394 respectivamente. No está de más señalar que, pese al sentido peyorativo y restrictivo del término "aculturación" desde la perspectiva antropológica, el DRAE define asépticamente el término como la "recepción y asimilación de elementos culturales de un grupo humano por parte de otro".

11 Notes towards the Definition of Culture, p. 59. 
Los tres aspectos esenciales del pensamiento sociocultural de Eliot señalados hasta ahora -el propio concepto de cultura, su nexo esencial con la religión y la tensión creativa entre unidad y diversidad- afectan también a sus planteamientos acerca de la construcción europea, una de las líneas de reflexión a las que más atención dedica.

Algunos de los ensayos más influyentes de su obra, en especial "Tradition and the Individual Talent" (1919) y "Dante" (1929)12, se ocupan de la reflexión acerca del pasado de Europa y de su situación contemporánea. En "Dante", por ejemplo, erige al poeta italiano en símbolo central de la unidad y de la tradición de la cultura europea y en referente para la transformación regeneradora que Eliot juzgaba necesaria para la supervivencia del continente. Su figura como poeta cristiano canónico le permite afirmar la relación de esencialidad entre la idiosincrasia cultural europea y el cristianismo. En la teoría cultural eliotiana, éste se configura como el elemento de cohesión de la diversidad cultural de Europa a lo largo de la historia. Frente al liberalismo y al comunismo, los dos grandes peligros que, según él, acechaban a la supervivencia de la tradición europea, Eliot veía en el cristianismo un contrapeso esencial. Para él, la tradición cultural y la democracia europeas sólo se entienden en el contexto cristiano. La simple sospecha de que Europa pueda abandonarlo u olvidar su importancia sitúa a Eliot en un escenario verdaderamente catastrofista, ya que, tal y como él ve el asunto, la sociedad que rompe con su tradición y con su religión es candidata a dejarse arrastrar por las masas y su pseudocultura y a convertirse en poco más que simple turba manipulable. ${ }^{13}$ Desde tal perspectiva, Europa, como comunidad de valores asentada en una identidad inequívocamente cristiana, se presentaba como elemento esencial de defensa frente a los impulsos nacionalistas, sectarios y totalitarios que la amenazaban de muerte. Y, desde luego, Eliot no aparece ni mucho menos aislado, sino que su ideario europeo cristiano se emparenta, por ejemplo, con el expresado por Churchill en su histórico discurso de 1946 y con el de intelectuales católicos como Benda, Bernanos o Mounier. ${ }^{14}$

El organismo espiritual y cultural de raíz cristiana que para Eliot constituye Europa está por encima de cualquier estructura política que, precisamente por ser política, es transitoria. Parece que no siempre se entiende o se acepta que la idea de Europa en la que Eliot cree y que defiende en sus escritos es eminentemente cultural -es decir, tiene que ver con la configuración, sobre todo a partir de los orígenes grecolatinos, de la identidad europea a lo largo de los siglos-, aunque no pueda evitar que su propia noción de cultura lo arrastre en ocasiones a las fronteras de la polí-

12 T. S. Eliot. Selected Essays. London: Faber and Faber, 1999 (1932), pp. 13-22 y 237-277 respectivamente.

13 T. S. Eliot. The Idea of a Chistian Society, p. 53.

14 Vid. E. San Miguel Pérez. Humanismo cristiano. Madrid: Centro de estudios Ramón Areces, 2005, p. 121-131. 
tica. Pero es en ese sentido preponderantemente cultural como convendría juzgar, por ejemplo, su afinidad, sin duda polémica, al ideario de Charles Maurras, el fundador de la ultraderechista Action Française, pues Eliot no escribe nunca como el político que no era, sino como crítico literario, social y cultural, algo que él mismo se encarga de subrayar en más de una ocasión. Ello hace que su pensamiento sociocultural, independientemente de la polémica que pueda causar, se perciba como más sutil cuando no abandona los terrenos de la cultura y que se vuelva más burdo en las pocas ocasiones en que se adentra en los peligrosos y traicioneros terrenos de la política.

La reflexión sobre la naturaleza y el futuro de la educación constituye otro de los elementos esenciales de la teoría sociocultural de Eliot, pues, en tanto que manifestación cultural de primer orden, le asigna una función capital en la configuración y desarrollo de una sociedad. De nuevo, más allá de que se discrepe o no de lo que Eliot plantea, lo cierto es que sus opiniones se asientan firmemente en lo que podría denominarse el "paradigma humanista" de la educación, que hunde sus raíces en la confluencia entre la paideia griega y la humanitas latina, por un lado, y la soteriología judeocristiana por otro $15 \mathrm{y}$, en tal sentido, se acercan mucho a determinadas posiciones del debate actual en torno a la función de la educación, a su crisis en la Europa de hoy en día y a los diferentes modelos educativos que se discuten. 16

El punto de partida de Eliot es, de nuevo, muy pesimista. Su análisis parte de la constatación de una situación que, a sus ojos, es casi catastrófica: según su criterio,

15 Vid. R. Carneiro. "Sociedad educativa y nuevo humanismo". En M. L. Amigo y M. Cuenca eds. Humanismo y valores. Bilbao: Universidad de Deusto, 2003, 389-404, p. 389.

16 No entra en el objetivo de estas páginas discutir con detenimiento un tema tan complejo y con tantas implicaciones como el de la educación en la sociedad actual. Sin embargo, interesa destacar, para ponerlas en relación de semejanza y de oposición al ideario eliotiano, algunas de las ideas que marcan el debate educativo contemporáneo desde una perspectiva humanista amplia. Así, el paradigma pedagógico humanista se define, en principio, en abierta oposición tanto al paradigma naturalista de raíz rousseauniana, centrado en los valores de la naturaleza y en el valor esencial de la espontaneidad del alumno frente a la autoridad del maestro, como al paradigma positivista, basado en el rechazo de la razón y de sus conocimientos en los dominios transcendentes, que han perdido objetividad y han cesado de ser fuente esencial de principios y de normas. (Vid. J. M. Quintana Cabanas. "Hitos para una pedagogía moderna". En M. L. Amigo ed. Humanismo para el siglo XXI. Bilbao: Universidad de Deusto, 2003, 261-267, pp. 263-264). Sobre dicho paradigma humanista amplio se asientan propuestas como el proyecto portugués de la Escuela Cultural (M. Ferreira. "El movimiento de la Escuela Cultural". En M. L. Amigo ed. Humanismo para el siglo XXI. Bilbao: Universidad de Deusto, 2003, 283-289), aunque conviene subrayar, para no olvidar la complejidad a veces contradictoria del asunto y la polisemia del propio concepto de humanismo, que también se reclama como humanista una propuesta educativa neomarxista como la de los Verdes alemanes, adscrita abiertamente al paradigma naturalista mencionado con anterioridad desde el momento en que sus postulados se basan precisamente en la "bondad originaria y total de la naturaleza humana" y en la "maduración del hombre (...) de modo espontáneo" (Vid. S. Uhl. "Las propuestas pedagógicas de los Verdes alemanes". En M. L. Amigo ed. Humanismo para el siglo XXI. Bilbao: Universidad de Deusto, 2003, 297-302, p. 298). 
la educación de su época había caído en las garras de un utilitarismo que, entre otras consecuencias, había relegado a un papel secundario, si no insignificante, a la cultura grecolatina, había arrinconado la importancia del mérito y rebajado los niveles de calidad y exigencia necesarios en una educación de calidad. ${ }^{17}$

Para contraatacar la ofensiva utilitarista, Eliot utiliza las armas más dogmáticas de su pensamiento: la vuelta a la ortodoxia y a la jerarquía de valores en general. De nuevo en este caso, su planteamiento de la educación excluye por completo cualquier atisbo de igualitarismo cultural. En este punto se hace necesario un análisis más fino de la teoría eliotiana, ya que no siempre se hace sencillo separar el elitismo de Eliot de su supuesta tendencia antidemocrática. Resulta útil y clarificadora en este sentido la puntualización de Vanheste, que distingue entre elitismo cultural y elitismo social. ${ }^{18}$ En buena medida, el rechazo e incluso la aversión que el ideario de Eliot pueda causarnos a los que nos acercamos hoy en día a sus escritos socioculturales es que no siempre encontramos separados con nitidez ambos elitismos. Vanheste ofrece como ejemplo contrario a Eliot el de Ortega y Gasset, para quien la aspiración a unos niveles culturales de calidad no implicaba el privilegio de grupos sociales concretos, aunque quizás cabría matizar que si bien "el intelectual Ortega aboga por la integración, el Ortega político exige que la masa sea dirigida hacia una educación lo más refinada posible". ${ }^{19}$ Sin embargo, a pesar de cierta evolución en su pensamiento, lo cierto es que Eliot abogó siempre por una igualdad relativa en la educación, una idea que él defendía acudiendo al argumento de que la ampliación democrática sin límites -por ejemplo mediante una extensión de la educación obligatoria o un acceso poco selectivo a las universidades- causaba una igualación por lo bajo de la exigencia y de la calidad que perjudicaba gravemente a la supervivencia del sistema educativo y, sobre todo, al mantenimiento de la alta cultura, uno de los objetivos irrenunciables de su ideario sociocultural. En cualquier caso, es de justicia reconocer también que sus ideas en torno al tema no se fosilizaron, sino que fueron evolucionando con el tiempo, y puede afirmarse que en la década de los 50 Eliot había interiorizado ya como concepto ético la igualdad de oportunidades educativas. ${ }^{20}$

\footnotetext{
17 Carlos García Gual no ha dejado de subrayar en múltiples ocasiones el efecto pernicioso que el declive del estudio de los clásicos grecolatinos tiene para el mundo contemporáneo. Vid. "El peso del pasado y la lectura de los clásicos". En M. L. Amigo y M. Cuenca eds. Humanismo y valores. Bilbao: Universidad de Deusto, 2003, 269-285 y "Del interés y la inactualidad de los clásicos". En M. L. Amigo ed. Humanismo para el siglo XXI. Bilbao: Universidad de Deusto, 2003, 291-296.

18 Op. cit., p. 294.

19 Van Ree. op. cit., p. 136.

20 R. Kojecky. op. cit., p. 205.
} 


\section{Eliot y la cuestión humanista}

Independientemente de las objeciones y reparos que con razón puedan planteársele, es conveniente subrayar que el pensamiento sociocultural de Eliot es fruto en buena medida de una profunda preocupación por el devenir y el buen estado de la sociedad. Su interés por los temas sociales se vio favorecido, sin duda, por la propia atmósfera familiar y cultural en la que se educó. La fe unitarista de su familia tenía uno de sus pilares básicos en las obras sociales y en la reverencia a unos ideales religiosos, comunitarios y educativos que adquirían todo su sentido gracias al bien general hacia el que se proyectaban. Su preocupación y análisis acerca de la situación cultural europea, la permanencia y validez de los clásicos, la función primordial de la educación o la importancia de la religión en la identidad cultural son cuestiones que, por su propia naturaleza, lo colocan en la tradición humanista europea. Y, sin embargo, pese a lo que en principio pueda afirmarse, la realidad que emana de sus escritos es que Eliot se situó siempre en una posición ambivalente y nunca llegó a comulgar del todo con el ideario humanista, pues sentía que en buena parte representaba ideas contrarias a las suyas, aunque tampoco dejaba de reconocer la afinidad que, en otros aspectos, lo ligaba a él. Grosso modo, puede sostenerse que, en general, sus discrepancias con el humanismo provienen en gran medida de su relación con la religión y con el lugar que ésta debe ocupar con respecto a aquél.

Su posición con respecto al humanismo se deduce de muchos de sus ensayos de crítica social, filosófica, literaria y religiosa, pero especialmente de tres de ellos, "The Humanism of Irving Babbitt", "Second Thoughts about Humanism" y "Religion without Humanism", publicados respectivamente en 1928, 1929 y 1930.21 Son fechas tempranas aún, pero en ellos se establece ya una posición intelectual que se mantuvo desde entonces prácticamente inalterada. El primer ensayo, como indica su título, se centra en las ideas del destacado humanista norteamericano Irving Babbitt (1865-1933), que ya desde sus años de estudiante en Harvard ejerció un magisterio considerable en Eliot, pero del que lo separó precisamente su discrepancia en torno a algunos conceptos definidores del humanismo.

Al contrario que Eliot, Babbitt, en obras como Rousseau and Romanticism (1919) y Democracy and Leadership (1924)22, aboga por un humanismo de raíz esencialmente secular, aunque ambos coinciden en subrayar la laxitud conceptual

\footnotetext{
21 "The Humanism of Irving Babbitt" y "Second Thoughts about Humanism" en Selected Essays, pp. 471-480 y 481-491 respectivamente; "Religion without Humanism" en N. Foerster ed. Humanism and America. Port Washington: Kennikat Press, 1967 (1930), pp. 105-112.

22 I. Babbitt. Rousseau and Romanticism. Indianapolis: Liberty Fund Inc., 1979 (1919); Democracy and Leadership. Whitefish: Kessinger Publishing, LLC, 2007 (1924).
} 
que afecta a la definición del mismo.23 Para Babbitt, son dos los rasgos que caracterizan el humanismo que él defiende: por una parte, la centralidad de lo humano frente a lo divino y, por otra, la recuperación del decoro, del término medio, que, según él, se había abandonado en el análisis de las preocupaciones e intereses del hombre. Detecta Babbitt que sectores importantes de la modernidad manifiestan un marcado rechazo a las posiciones del humanismo; pese a ello, su obra está dedicada en buena medida a subrayar la importancia del ideario humanista para el mundo contemporáneo y a contrarrestar la idea errónea, según él, de que el humanismo se refugia en los asuntos del pasado para no afrontar los problemas del presente. Precisamente este segundo objetivo de Babbitt coincide en gran medida con la idea axial de Eliot en torno a la tradición y al papel esencial del pasado para la configuración del presente. Ambos coinciden en ver la tradición no como algo inerte sino como una fuerza viva que consigue suspender el paso del tiempo y crear una atemporalidad en la que pasado, presente y futuro se interrelacionan creativamente. ${ }^{24}$

De la contundencia con la que Babbitt defiende la naturaleza secular que para él debe caracterizar al humanismo no se desprende, en absoluto, un rechazo tajante al papel que pueda cumplir la religión. La lectura del ensayo de Eliot conduce a pensar que éste no entiende del todo -o no quiere entender- que la argumentación de Babbitt no es, ni mucho menos, antirreligiosa, sino que, al contrario, subraya que la cooperación entre el humanismo y el cristianismo, además de necesaria, puede ser muy fructífera. En este punto, la centralidad que en su teoría sociocultural tiene la religión lleva a Eliot a afirmar la inevitable subordinación a ésta del humanismo. En cambio, la firme apología que Babbitt hace de la autonomía del humanismo se basa en el convencimiento de que sus principios han demostrado su validez para afrontar por sí solos los desafíos del mundo moderno. Ello no le impide reconocer lo mucho que el humanismo se beneficia de un contexto de cooperación con la religión. Pese a la interpretación errónea de Eliot, del ideario de Babbitt se deduce con facilidad que éste considera un error suponer que el humanismo pueda cumplir la función que corresponde a la religión. Es más, se muestra convencido de que es más factible que la religión funcione sin el humanismo que al contrario.

En resumen, del ideario de Babbitt se desprende una actitud tolerante y optimista ante la relación que deben establecer la religión y el humanismo. En su pensamiento, éste se configura como un campo abierto a la cooperación social e inte-

\footnotetext{
23 Una definición que continúa siendo problemática. Para un repaso a la configuración histórica del carácter polisémico del concepto, vid. F. Duque. "Del descrédito del humanismo por saturación de proclamas humanistas". En M. L. Amigo y M. Cabezas eds. Humanismo y valores. Bilbao: Universidad de Deusto, 2003, 405-436.

${ }^{24}$ En buena medida, tanto Eliot como Babbitt entroncan con la tradición del humanismo renacentista, claro ejemplo para García Gual del "valor de una tradición, redescubierta y valorada contra otra, más próxima, pero sentida como oscura y opresiva. Volver a paradigmas del pasado puede ser un avance hacia la libertad" (“El peso del pasado y la lectura de los clásicos”, p. 274).
} 
rreligiosa, un rasgo éste que, en el contexto de conflictividad religiosa y cultural que está caracterizando los primeros años del siglo XXI, demuestra la pertinencia en la actualidad de las ideas humanistas de Babbitt en torno a la mesura y al sentido común que deben presidir los contactos entre religiones y culturas.

La cooperación posible e incluso necesaria entre el humanismo y la religión la descarta Babbitt a la hora de enjuiciar el naturalismo o humanitarismo (humanitarianism), como él también lo denomina. En este punto, en el que coincide con Eliot, destaca su furibundo ataque a Rousseau. Babbitt considera especialmente errónea la habitual vinculación del filósofo ginebrino con los ideales del humanismo. Muy al contrario, desde su punto de vista, la filosofía rousseauniana atacó con contundencia los principios del humanismo y la religión tradicionales e inauguró el periodo naturalista en el que, según él, todavía estaba inmersa la cultura europea. Además, las creencias del humanitarismo habían favorecido la extensión del carácter temperamental propio del romanticismo en perjuicio del decoro y la mesura humanistas y habían extendido la falsedad acerca de la omnipotencia del hombre y de la ciencia sobre la naturaleza. La bestia negra de Babbitt en este sentido es la filosofía baconiana, que, a su juicio, dio carta blanca al descomunal poder de la ciencia y de la tecnología y, con ello, a los abusos del pensamiento materialista y a la preponderancia del utilitarismo sobre los valores trascendentes. ${ }^{25}$ Los principios humanistas de Babbitt lo sitúan no precisamente como un enemigo de la ciencia como tal sino de los abusos cometidos en su nombre y de su inclinación a extralimitarse y a invadir terrenos que no le corresponden. ${ }^{26}$ Tanto Babbitt como Eliot coinciden, por un lado, en denunciar cualquier tendencia naturalista que intente postularse como sustituta de la religión o del humanismo y, por otro, en subrayar la diferencia esencial entre el humanismo y el naturalismo. Dicha diferencia estriba en que el objetivo central del humanista es poder referir la variedad de la experiencia a un centro proporcionado por la conciencia de la tradición, una conciencia que hace posible que el ser humano observe la esencia permanente de la naturaleza que lo liga al resto de la humanidad.

\footnotetext{
${ }^{25}$ Sin duda, Babbitt habría compartido la tajante afirmación de Erwin Schrödinger, premio Nobel de Física en 1933, el mismo año de la muerte de Babbitt, de que "lo peor es que el tremendo desarrollo material produjo una perspectiva materialista”. E. Schrödinger. Ciencia y humanismo. Barcelona: Tusquets, 1998. El ensayo de Schrödinger proviene de cuatro conferencias impartidas en 1959 bajo el significativo título de "La ciencia como parte integrante del humanismo".

26 A pesar del amigable título del ciclo de conferencias de Schrödinger, lo innegable es que las relaciones entre ciencia y humanismo, como dominios enfrentados, no han sido precisamente fáciles en un periodo, el siglo XX, en el que la ciencia ha perdido la inocencia y en el que, siguiendo a Habermas, la técnica se ha erigido en ideología (J. Habermas. Ciencia y técnica como ideología. Madrid: Tecnos, 2009). Vid. F. J. Gil. “Tecnología y esfera pública en Jürgen Habermas”. Revista CTS, 5, 2, 141-152; D. Gracia. "Ciencia y ética". En VV.AA. Humanismo y ciencia. La Coruña: Fundación Paideia-Casa de las Ciencias, 1994; y M. Lorente. "Ciencia y humanismo". En M. L. Amigo ed. Humanismo para el siglo XXI. Bilbao: Universidad de Deusto, 2003, 143-150.
} 
Más allá de las coincidencias con Babbitt, el pensamiento de Eliot está determinado por la consideración esencial de la religión, y esto, irremediablemente, acaba afectando a su posición intelectual con respecto al humanismo en un asunto determinante: la primacía o no de la dimensión humana. Cualquier discusión en torno al asunto no puede obviar que, en cualquier caso, el humanismo de Eliot es esencialmente dependiente de la religión y que, como tal, nunca podrá aspirar a erigirse en sustituto de la misma. Sus reticencias con respecto al humanismo secular derivan precisamente del miedo a que éste acabe transformado en un sistema filosófico dogmático. Ese temor parece estar en todo momento detrás de la lectura un tanto interesada que hace Eliot de las ideas de Babbitt con el propósito de sostener su tesis fundamental en torno al tema, es decir, que el humanismo carece casi totalmente de sentido si no es en dependencia de la religión. La cautela intelectual de Eliot tiene que ver en gran medida con la debilidad que él detecta en los límites entre el humanitarismo o naturalismo tan denostado también por Babbitt y el humanismo secular: a su juicio, mientras que para el primero la dimensión animal es primordial en detrimento de la humana, el segundo, al caer en la "falacia humanística" de la que habla Hillman, ${ }^{27}$ privilegia casi exclusivamente lo humano frente a lo divino y, desde su perspectiva, puede degenerar con facilidad y caer en el naturalismo. En ningún momento, sin embargo, comete Eliot el error de negarle al humanismo su papel histórico ni la importancia de sus ideas para el mundo moderno, pero sí rechaza tajantemente que pueda equipararse esa labor a la función determinante del cristianismo en la configuración histórica de la cultura europea.

El carácter marcadamente antisubjetivo del pensamiento de Eliot lo lleva a rechazar precisamente el subjetivismo que, a su juicio, determina de manera irremediable, junto a su naturaleza secular, el pensamiento humanista de Babbitt, hasta el punto de anularlo. Como ha quedado señalado, Eliot realiza una lectura algo sesgada de las ideas de Babbitt con el objetivo de subrayar exclusivamente la autonomía del humanismo que éste defiende y obviar su reconocimiento de la fructífera influencia que la religión puede y debe ejercer sobre el mismo. En buena medi$\mathrm{da}$, le interesa partir de la supuesta defensa -falsa, en realidad- que Babbitt realiza de un humanismo sin religión para, desde ese presupuesto, atacar el concepto del autocontrol sobre el que, según él, se sostiene la teoría humanista de Babbitt. En este sentido, puesto que, desde una perspectiva exclusivamente política, la evolución de la democracia implica necesariamente el debilitamiento progresivo e incluso la desaparición de la restricción ejercida por instituciones externas como el sistema de clases sociales, la aristocracia o la monarquía, Eliot admite que el hombre deba dotarse de un autocontrol que las reemplace. Sin embargo, en el terreno religioso, por más que la institución eclesiástica y su ritual también se debiliten en la

27 J. Hillman. Re-imaginar la psicología. Madrid: Siruela, 1999. Cit. en L. Garagalza. "Hermenéutica del lenguaje y simbolismo". Endoxa, 20, 2005, 245-261, p. 258-259. 
sociedad moderna, no puede admitir que el individuo pueda sustituir el dogma ético de la religión por un autocontrol espiritual -"inner check" en la terminología de Babbitt- que, a su juicio, implicaría el triunfo de un subjetivismo culturalmente destructivo.

En "Second Thoughts about Humanism", Eliot vuelve a incidir en las ideas desarrolladas en el ensayo de 1928, aunque en esta ocasión el objetivo de su ataque, mucho más furibundo e incisivo que el anterior, es el conocido como "Nuevo Humanismo", que él focaliza en la figura de Norman Foerster (1887-1972), lo cual no fue óbice para que en 1930 publicara el ya citado "Religion without Humanism" en el libro Humanism and America editado por el propio Foerster. Su argumentación se basa una vez más en el, a su juicio, insuficiente papel asignado a la religión en el debate en torno al humanismo y, en concreto, en el extremismo ideológico neohumanista que para él supone hacer de los factores y circunstancias humanas la medida de todas las cosas. Para construir su armazón argumentativa, Eliot recurre a las razones con las que T. E. Hulme en sus Especulaciones justifica su rechazo del humanismo y que apuntan esencialmente a la ética relativista a la que conduce el subjetivismo humano cuando prescinde en sus consideraciones de la dimensión divina. Así, la idea central sobre la que para él se sostiene el edificio ideológico del humanismo moderno - la perfectibilidad sin límites del ser humano- choca de frente con su propia creencia -que comparte con Hulme- en un absoluto (la cursiva es de Eliot) al que el hombre nunca (ídem) podrá llegar. 28

Tanto en "The Humanism of Irving Babbitt" como en "Second Thoughts about Humanism", Eliot focaliza preferentemente sus reflexiones en la dirección del humanismo a la religión. Sin embargo, en el ensayo de 1930 "Religion without Humanism" queda claro desde el propio título que su objetivo es moverse en la dirección contraria, la que lleva desde la religión al humanismo, para así incidir también en los beneficios que ésta obtiene de una relación de colaboración -no de dependencia- con el ideario humanista. Para ello retoma las funciones mencionadas anteriormente y asigna al humanismo un papel importante como moderador de las creencias y prácticas religiosas y como protección crítica contra posibles derivas fundamentalistas o fanáticas. ${ }^{29}$ Es cierto que para Eliot el gran defecto del huma-

28 "Second Thoughts about Humanism", p. 490. La filosofía de Hulme moldea de modo importante el pensamiento de Eliot, pues su aversión a la idea naturalista de la bondad innata del hombre encuentra firme apoyo en el ataque a la filosofía de Rousseau por parte de Hulme, para el que el humanismo o humanitarismo que subyace en las tendencias románticas es una herejía, la adopción errónea de conceptos falsos. La ceguera romántica ante la naturaleza limitada del hombre conduce a una degradación tanto de lo humano como de lo divino. De las ideas de Hulme deriva Eliot su definición del romanticismo como "exceso". Vid. R. Schuchard. "Eliot and Hulme in 1916". PMLA, 88, 4, 1083-1094, p. 1088.

${ }^{29}$ Aunque en 1933 todavía habría de afirmar que el interés de humanistas como Babbitt por la filosofía oriental suponía un desarraigo de la tradición cristiana, en 1940-1941 Eliot llegó a admitir lo 
nismo es no poseer ni la disciplina intelectual propia de la filosofía y la ciencia ni la disciplina emocional que sólo puede aportar la religión; pero no es menos cierto que su reflexión sobre el tema incide especialmente en que, sin la ayuda auxiliar del humanismo, la religión corre el riesgo de petrificarse, y la ciencia, de convertirse en un aséptico conjunto de técnicas que acabe derivando en aberraciones. El humanismo adquiere así finalmente en la teoría sociocultural de Eliot una importante función ligada inexorablemente a la religión, una dependencia mutua, al fin y al cabo, que de múltiples modos mantiene su vigencia -incluso para los que se acercan al asunto desde posiciones no religiosas- en bastantes de las propuestas humanistas de la era de la globalización. ${ }^{30}$

Como ha quedado apuntado, de "Religion without Humanism" se deduce una posición más abierta de Eliot a aceptar la importante función que debe desempeñar el humanismo en ese concepto amplio de cultura determinado desde su raíz misma por el papel central de la religión. En este punto, conviene subrayar la gran importancia del humanismo neotomista de Jacques Maritain (1882-1973), sobre todo de su célebre Humanisme intégral (1936) 31 , para la configuración del ideario humanista eliotiano.

Pese a la ambigüedad que reconoce en el término y al uso interesado que de él pueda hacerse, Maritain parte de la asunción inicial de que el humanismo es una cuestión inseparable de la cultura, que en su pensamiento adquiere unos rasgos de indudable paralelismo con el concepto eliotiano visto hasta ahora. En efecto, para Maritain la cultura aparece como una actividad global propiamente humana definida por lo que él denomina el "polo económico", es decir, por la satisfacción de las necesidades materiales inherentes a la vida humana, y, sobre todo, por el papel esencial reservado a la religión -el polo religioso- en el desarrollo moral del hombre. En la filosofía de Maritain, el humanismo integral supone una realización social y temporal, es decir, una encarnación -pues tal es el término que utiliza- de la atención a lo humano inherente al orden espiritual y encaminada al objetivo de una comunidad fraterna. La trascendencia desde lo humano -la cultura- a lo espiritual y la encarnación de lo espiritual en lo humano es, tanto para Maritain como para Eliot, un rasgo definidor irrenunciable de su concepción humanista, que se distingue así con claridad de otras concepciones del humanismo, y se sitúa radicalmente en el extremo opuesto de cualquier teoría naturalista o humanitarista. En tal sentido, Maritain -y con él el propio Eliot- asienta las bases de su humanismo integral en fuentes clásicas -muy señaladamente Aristóteles- y cristianas, para las que el sentido del mundo

mucho que podría aprender un cristianismo en decadencia del pensamiento oriental. Vid. P. Murray. T. S. Eliot and Mysticism. New York: St. Martin Press, 1991, p. 127.

30 Vid. Camps y Valcárcel. op. cit. y J. M. Mardones. "Religión y humanismo. La religión en la emergencia de una cultura global". En M. L. Amigo ed. Humanismo para el siglo XXI. Bilbao: Universidad de Deusto, 2003, 445-452.

31 J. Maritain. Humanisme intégral. Paris: Aubier, 2000 (1936). 
se explica en su origen por la trascendencia. Frente al humanismo de Babbitt -religioso sólo en cuanto que abierto a la cooperación e influencia determinante de la religión, pero esencial y primordialmente secular-, el humanismo de Maritain y Eliot es religioso en tanto que es teocéntrico. Las concomitancias con el pensamiento de Eliot son también evidentes en el rechazo al atropocentrismo naturalista que, a juicio de ambos, ha querido hacer del impulso humano el origen absoluto del mundo. Para Maritain Nietzsche se erige en la culminación de una cadena de hitos naturalistas que incluye, entre otros, a Comte, Hegel y, de manera muy señalada, a Rousseau, a quien atribuye la formulación de la "teología naturalista absoluta", un rechazo del pensamiento rousseauniano que lo une a Eliot y, como se ha visto, también a un humanista secular pero profundamente antinaturalista como Babbitt. El contexto histórico europeo del momento explica que Maritain presente su humanismo integral como antídoto de las corrientes naturalistas y, muy particularmente, del "humanitarismo marxista". A su juicio, la evolución del humanismo antropocéntrico había desembocado en un enfrentamiento entre dos posiciones puras: la atea y la cristiana. Frente a la deshumanización y a la exclusión de toda la herencia cristiana que, a su juicio, se deriva del ateísmo, su propuesta humanista cristiana se asienta en un carácter integrador que lo lleva, por ejemplo, a valorar -más allá de sus obvias discrepancias ideológicas- las aportaciones del ideario socialista o a reivindicar la tolerancia civil propugnada por Voltaire y la rebeldía anticonformista de Lutero como contribuciones de calado al crecimiento de la dignidad humana. El argumento de Maritain es que su noción de cultura humanista cristiana es capaz de integrar a Voltaire o Lutero, frente a un sistema marxista que, ya de entrada, excluye a San Agustín o a Santa Teresa.

En el humanismo integral de Maritain encontró Eliot una propuesta que casaba muy bien con sus ideas en torno al concepto de cultura, al papel del humanismo y a la centralidad de la religión. Además, cabría pensar con mucho convencimiento que la tolerancia y apertura dogmática que caracteriza la obra del filósofo francés sirvieron también para atemperar en mayor o menor grado las posiciones más radicales de Eliot.

\section{Conclusión}

El acercamiento actual al pensamiento sociocultural de T. S. Eliot produce un efecto ambiguo. Por un lado, el enjuiciamiento crítico de parte importante de sus presupuestos conduce a pensar que estamos ante una teoría con evidentes tintes de utopía social, incluso ya en la época en que se planteó. Además, desde la perspectiva de las sociedades democráticas europeas, no deja de ser cierto que algunos de los planteamientos de Eliot -en particular sus propuestas sobre el mantenimiento de las 
clases sociales, el papel de las élites o el antiigualitarismo educativo- son cuanto menos polémicos e incluso muy ajenos al sentimiento mayoritario de la opinión pública actual. Sin embargo, la preponderancia que en las últimas décadas han adquirido los argumentos ideológicos en la consideración global de su obra no debe hacer olvidar que la teoría sociocultural de Eliot nace de un contexto histórico muy concreto y de unas posiciones ideológicas de marcado carácter conservador. Pero su conservadurismo sociocultural, político y religioso -que, por otro lado, era común en mayor o menor medida a muchos otros intelectuales del momento- no justifica la asociación que, a la ligera, pueda hacerse, por ejemplo, con el ideario fascista. Independientemente de ciertas veleidades, de determinadas declaraciones poco afortunadas o de su admiración por Maurras, ver en Eliot a un antidemócrata, defensor cerrado del fascismo, es no entender que, por ejemplo, su defensa de la alta cultura y hasta el concepto mismo de cultura que defiende -se esté o no de acuerdo con él- es incompatible en su esencia con la ideología fascista. De igual modo, su rechazo del materialismo derivado del naturalismo y los reparos ante un humanismo excesivamente centrado en lo humano son incompatibles con el ideario fascista, en tanto que, como otros totalitarismos del siglo XX, el fascismo hace de la revolución industrial y del pensamiento científico el fundamento de su poder social. ${ }^{32}$

Más allá de lo polémicos y incluso anacrónicos que puedan resultar hoy sus escritos socioculturales en algunos de sus aspectos, resulta de justicia reconocer que buena parte de los temas a lo que Eliot dedicó su análisis continúan en el centro del debate intelectual europeo: la construcción de Europa como un ente cultural y político y no como una mera zona de librecambismo; las tensiones nacionalistas y regionalistas; el contacto no siempre fácil entre unos valores cristianos tradicionalmente dominantes y los de otras religiones; el papel de la ciencia; la aguda crisis de los modelos educativos y, en concreto, de las humanidades; o la propia articulación de la democracia.

El asunto del humanismo se revela precisamente como una de las claves para valorar la actualidad de los planteamientos eliotianos y la necesidad, por tanto, de reconsiderarlos con objetividad. Lo que se deduce del análisis de la posición conflictiva que Eliot mantuvo a lo largo de su vida con los presupuestos humanistas va más allá de la función esencial que, como ha quedado señalado, le asigna a la religión. En realidad, la cuestión de la filiación humanista de Eliot sirve para rescatar un debate intelectual de altura que implicó a personalidades tan influyentes como Babbitt y Maritain, y que por derecho propio se inscribe en la gran y poliédrica discusión que a lo largo del siglo XX y en estos inicios del XXI intenta responder, también desde posiciones abiertamente antihumanistas, a la célebre pregunta de Jean Beaufret a Heidegger: "Comment redonner un sens au mot 'humanisme"”.

32 Vid. J. A. Estrada. "El humanismo en el siglo XXI". En M. L. Amigo ed. Humanismo para el siglo XXI. Bilbao: Universidad de Deusto, 2003, 61-67, p. 62. 
Divergencias aparte, intelectuales como Babbitt, Maritain, Ortega, Benda y otros compartían con Eliot una profunda preocupación por asuntos intangibles a veces pero determinantes para la sociedad. Para todos ellos, el humanismo se presenta como un antídoto contra los excesos del materialismo y del utilitarismo. Para Eliot, las ideas de un humanismo ligado siempre a la centralidad de la religión evita la fosilización dogmática de ésta, aparte de cumplir una determinante función mediadora en el diálogo intercultural e interreligioso. En cualquier caso, en una época -la actual- necesitada, según la propuesta de Llano, de un "humanismo cívico" 33 y determinada, según Bobbio, por un progreso científico y técnico "cada vez más rápido, cada vez más imparable, cada vez más irreversible" 34 , y en la que la globalización, Internet, las migraciones y otros muchos factores están redefiniendo el concepto de identidad, la noción de cultura o el valor de la educación, resulta enriquecedor rescatar debates como el del humanismo aquí tratado, que, desde su lejanía temporal, vuelve a poner de relieve la idea de que el presente sólo se construye desde la conciencia activa del pasado, una idea que Eliot resumió con certeza maestra en los primerísimos versos de Four Quartets: "Time present and time past / Are both perhaps present in time future / And time future contained in time past".

Pablo Zambrano Carballo

Departamento de Filologías Integradas

Universidad de Huelva

zambrano@uhu.es

\footnotetext{
33 A. Llanos. Humanismo cívico. Barcelona: Ariel, 1999.

34 N. Bobbio. "Demasiadas dudas en la izquierda". EL PAÍS (24-4-1993). Cit. en R. Mate. "Ciencia, religion y filosofía". En VV. AA. Humanismo y ciencia. La Coruña: Fundación Paideia-Casa de las Ciencias, 1994, 33-49, p. 33.
} 